\title{
Dual-Band Patch Antenna with Filtering Performance and Harmonic Suppression
}

\author{
Chun Xu Mao, Steven Gao, Yi Wang, Benito Sanz-Izquierdo, \\ Zhengpeng Wang, Fan Qin, Qing Xin Chu, Jianzhou Li, Gao \\ Wei, Jiadong Xu
}

\begin{abstract}
A novel design of dual-band antenna with integrated filtering performance is proposed. A low-profile aperture-coupled U-slot patch antenna is employed for dual-band operation with a uniform polarization, which is fed by a dual-mode stub-loaded resonator (SLR). The U-slot patch works as a dual-mode resonator of the dual-band filter as well as the radiation element. The odd- and even-mode of the SLR are coupled and tuned with the U-slot patch, generatif two $2^{\text {nd }}$-order operation bands at 3.6 and 5.2 GHz. Comparea ith the traditional patch antenna, the proposed antenna exhibils mproyed bandwidth and frequency selectivity. In addition, the log alyidths can be controlled by adjusting the coupling strengtryeen the SLR and the patch. Furthermore, the higher-order hap norics can be suppressed over a broadband without increasing he igotprint of the design. Measured and simulated results agre well with each other, showing an excellent performance in tops of impedance matching, bandwidths, $2^{\text {nd }}$-order filtering, $t$-of-band rejection, cross polarization discrimination (XPD) and gain 1 th poth bands.

Index Terms - Dual-band, bandwidth, $2^{\text {nd }}$-ord r, Hiltering, U-slot patch, harmonic suppression.

\section{INTRODUCTION}

MODERN wireless communication systems demand RF front-ends with features of compactness, highly integration, multiband and multifunction. Patch antenna is considered as one of the most widely used antennas owing to its low profile, low cost, unidirectional and compatibility with backend circuits. Significant effort has gone into designing dual-band or multi-band patch antennas [1]-[4]. Dual-band operation can be obtained by manipulating the fundamental mode and higher order modes of the patch [5], [6].

To reduce the volume and complexity of RF front-ends and meet the demand for multifunctional operation, integrated passive devices such as power dividers, filters and antennas have been considered as a promising solution. The integration has the added benefits of improving the frequency response and removing the $50 \Omega$ interconnections and matching networks [8]. In [9], multiplexers were proposed based solely on

This manuscript is submitted on October 31, 2015. This work is supported by the project "DIFFERENT" funded by EC FP7 (grant no. 6069923). YW is supported by UK EPSRC under Contract EP/M013529/1.

C. X. Mao, S. Gao, and B. Sanz are with the School of Engineering and Digital Arts, University of Kent, UK. (e-mail: cm688@kent.ac.uk, s. gao@kent.ac.uk).

Y. Wang is with the Department of Engineering Science, University of Greenwich, UK. (e-mail: yi.wang@greenwich.ac.uk).

Z. P. Wang is with the School of Electronic and Information Engineering, Beihang University, China (e-mail: wangzp@buaa.edu.cn).

Q. X. Chu is with South China University of Technology, China (e-mail: qxchu@scut.edu.cn).

F. Qin, J. Li, G. Wei and J. Xu are with Northwestern Polytechnic University, China.

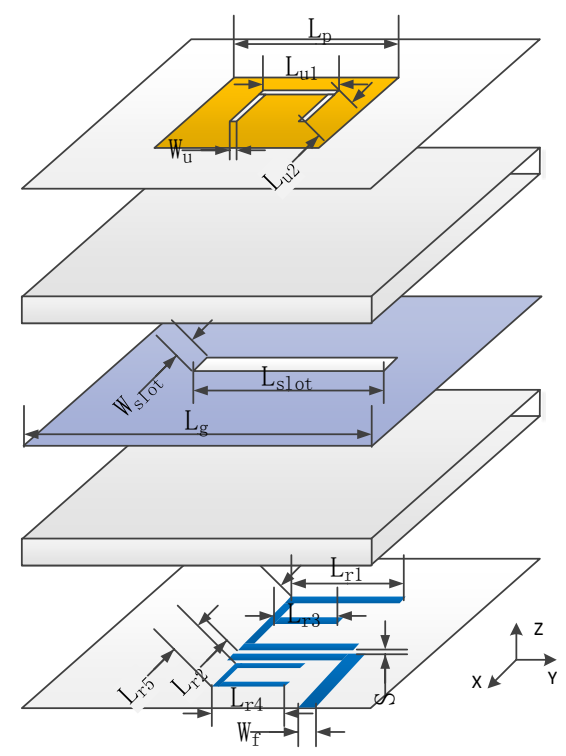

(a)

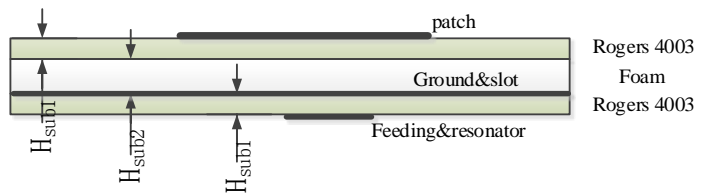

(b)

Fig.1. Configuration of the proposed dual-band filtering antenna: (a) exploded perspective view, (b) the stacked structure.

resonators to eliminate traditional transmission-line based Gistribution networks. The integration of filter and antenna has o. vn advantages such as reduction of RF front-end [10], mo bandwidth and frequency selectivity [11] and han nic suppression [12]-[13]. Nevertheless, many challeng semain in the design and optimization of filtering antennas as multiband operation and tunable bandwidth. To the bes author's knowledge, the problems haven't been fully consuderey and solved. In [14] - [15], two dual-band filtering patch anternas were proposed. However, the rectangular patch in 14 wrks in two orthogonal polarizations at the two bands and complicated matching structures were needed. Besides, the eak gains were only $-1.8 \mathrm{dBi}$ and $1.1 \mathrm{dBi}$ at the two bands and uny unted harmonics appeared between the two bands. 15 , the $\mathrm{TM}_{10}$ and $\mathrm{TM}_{30}$ modes of the patch were employet, the gain was only -4 $\mathrm{dBi}$ and the feeding network was also complex. In [16], a tri-band filtering antenna was proposed by cascading a triband filtering with a wideband antenna.

In this communication, a novel dual-band filtering antenna with broadband harmonic suppression performance is proposed for 3.6/5.2 GHz WiMAX and WLAN applications. A U-slot patch antenna is integrated with a dual-mode stub loaded resonator (SLR) through electromagnetic coupling, producing a $2^{\text {nd }}$-order dual-band filtering antenna. Compared with tradition patch, the bandwidth, filtering and harmonics suppression are significantly improved. In addition, the bandwidths of the antenna can be adjusted in the designing process. Both the simulated and measured results exhibit improved bandwidth and radiation performances. 


\section{ANTENNA DESIGN}

\section{A. Configuration}

Fig. 1 shows the configuration of the proposed dual-band filtering antenna. The design is a stacked structure composed of two substrates separated by a thin foam $(1 \mathrm{~mm})$. The square patch is printed on the upper layer of the top substrate with a U-shaped slot is etched in it for dual-band operation. The mechanism and the design method has been detailed in [3]. The feeding line and the dual-mode E-shaped SLR are printed on the bottom layer of the lower substrate. The patch and the feeding network share a same ground plane in the middle layer with an aperture etched in it. Fig. 1(b) illustrates the stacked structure of the proposed design. Rogers 4003 substrate with a dielectric constant of 3.55 and a loss tangent of 0.0027 was used. All simulations performed using High Frequency Simulation Software (H'SS15) and the optimized parameters are listed in Table I.

\section{B. Stub-Loaded Resonator}

To achieve the dual-band pachantenna with filtering performance, a dual-mode SLR is enployed to couple and synthetically tune with the U-slot patch traditional method of cascading a filter with an antenna [12], the filtering and radiating components a seamlessly integrated in this work. The U-slot patch here vos as the last-order dual-mode resonator of the $1^{\text {st }}$-ordendy and passband filter (SLR) as well as the radiating elem nt. contributes to a higher order filtering performance ( $2^{\text {nd }}-\mathrm{Cr}$ ) while maintaining a compact footprint.

Owing to the high freedom in controlling the modes, the E-shaped SLR is widely used as a dual-mode resonator in filter design. The SLR can be analyzed using the odd- and even-mode method, as previously detailed in [15]-[17]. When the odd-mode is excited, the center part of the SLR is equivalent to a shorted end and the resonant frequency can be approximately derived as,

$$
f_{\text {odd }}=\frac{c}{2 \sqrt{\varepsilon_{r}}\left(2 L_{r 1}+L_{r 2}\right)}
$$

When the even-mode is excited, the symmetrical plane can be viewed as an opened end and the resonant frequency can be expressed as,

$$
f_{\text {even }}=\frac{c}{2 \sqrt{\varepsilon_{r}}\left(L_{r 1}+L_{r 2} / 2+L_{r 3}\right)}
$$

where the $f_{\text {odd }}$ and $f_{\text {even }}$ are the odd- and even-mode resonant frequencies of the SLR. $c$ is the light velocity in free space and $\varepsilon_{r}$ is the effective permittivity. Thus, the odd-mode and even-mode resonant frequencies can be tuned easily by adjusting the dimensions of the SLR.

TABLE I

PARAMETERS OF THE PROPOSED ANTENNA: (MM)

\begin{tabular}{cccccccc}
\hline \hline $\mathrm{L}_{\mathrm{p}}$ & $\mathrm{L}_{\mathrm{u} 1}$ & $\mathrm{~L}_{\mathrm{u} 2}$ & $\mathrm{~W}_{\mathrm{u}}$ & $\mathrm{L}_{\mathrm{g}}$ & $\mathrm{L}_{\text {slot }}$ & $\mathrm{W}_{\text {slot }}$ & $\mathrm{L}_{\mathrm{r} 1}$ \\
27.2 & 7.5 & 8 & 0.45 & 60 & 9.4 & 0.7 & 8.2 \\
\hline $\mathrm{L}_{\mathrm{r} 2}$ & $\mathrm{~L}_{\mathrm{r} 3}$ & $\mathrm{~L}_{\mathrm{r} 4}$ & $\mathrm{~L}_{\mathrm{r} 5}$ & $\mathrm{~S}$ & $\mathrm{~W}_{\mathrm{f}}$ & $\mathrm{H}_{\text {sub1 }}$ & $\mathrm{H}_{\text {sub2 }}$ \\
8 & 6.15 & 4.2 & 2.8 & 0.3 & 1.8 & 0.813 & 1 \\
\hline \hline
\end{tabular}

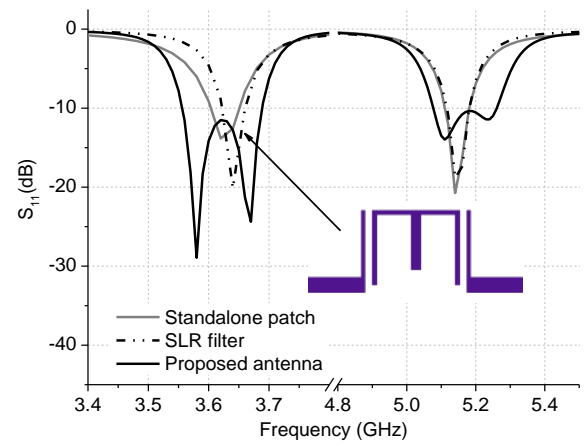

Fig. 2. The $S_{11}$ comparison among standalone U-slot patch, SLR filter and proposed filtering antenna.

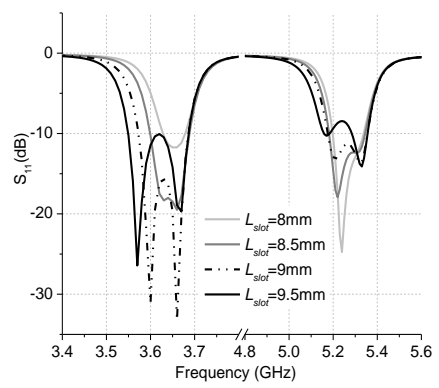

(a)

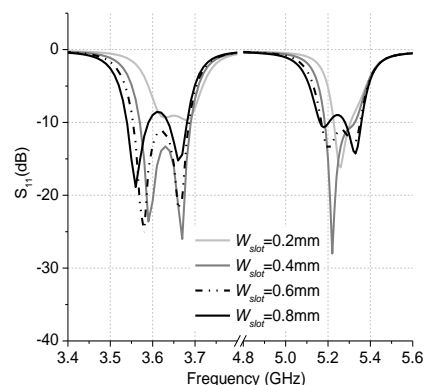

(b)
Fig. 3. The variation of bandwidth with different: (a) $L_{\text {slot }}$, (b) $W_{\text {slot }}$.

\section{Improved Frequency Response}

The bandwidth of the dual-band U-slot patch antenna is usually narrow, especially when the profile is low. Methods sych as increasing the profile of the design or loading the parasitic are commonly used to increase the bandwidth at the e. ense of a higher profile. In this design, a new method for enlang the two bandwidths of the patch antenna is presented $y$ using a dual-mode resonator to feed a U-slot patch electromagnetic coupling, higher-order frequency roses at the two bands can be achieved without increasing the pripe of the antenna.

Fig. 2 compars th simulated $S_{11}$ of standalone U-slot patch, SLR filter and the prosed filtering antenna. It is observed that the proposed antennare 10 its improved bandwidths of $4 \%$ at both bands with $2^{\text {nd }}$-ordd ling performance. In each band, two reflection zeros can beig to sharp roll-offs at both bands. In contrag, the traditional U-slot patch only shows one pole in each ban. an the bandwidths at both
bands are only about $1.8 \%$.

\section{Tunable Bandwidth}

Bandwidth is one of the key issues to be concerned in filtering antenna design. In bandpass filter design, the bandwidth can be tuned by adjusting the coupling strength between the resonators [16]. In our previous work [17], similar approach was adopted to tune the bandwidth of the antenna.

Fig. 3(a)-(b) shows the bandwidths of the two operation bands with different lengths and widths of the aperture. It is observed that when the length of aperture $L_{\text {slot }}$ is shorter than 8 $\mathrm{mm}$, the two reflection zeros merge together, leading to a narrow bandwidth of less than $50 \mathrm{MHz}(\mathrm{FBW}=1.4 \%)$. As $L_{\text {slot }}$ increases, indicating that the coupling strength between the SLR and the U-slot patch increases, the two reflection zeros are divided and a wider bandwidth of over $150 \mathrm{MHz}(4.2 \%)$ is 


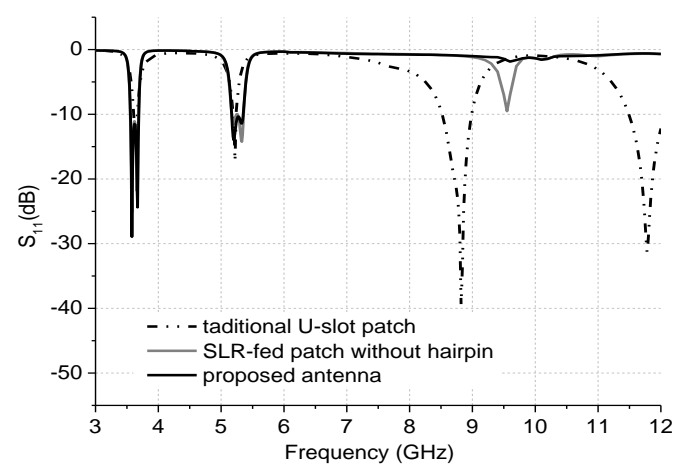

Fig. 4. The harmonic response of traditional U-slot patch, SLR-fed patch without hairpin and proposed filtering antenna over a wideband.

achieved for the low-band. The length of the aperture has a similar but less signitat effect on the high-band operation. The effects of the width the aperture on the bandwidth are shown in Fig. 3(b). Whent Kigh of the aperture decreases from 0.8 to $0.2 \mathrm{~mm}$, the bund ans of the low-band and high-band decrease from $4.2 \%$ to 8 and from $3.8 \%$ to $1.2 \%$, respectively. Different from the dujional patch antenna, where the bandwidth is usually tuned ad ysting the profile of the antenna, this work provides a new hod to tune the bandwidth of the antenna while keeping the file of the
antenna unchanged.

\section{E. Harmonic Suppression}

Harmonics is a serious problem to be concerned in communication systems. Traditionally, harmonics are eliminated by cascading a filter at the backend [12]. Howe r this increases the complexity and the volume of the RF front-end. In this integrated filtering antenna, harmonic suppression is taken into consideration. Fig. 4 shows the harmonic response of a traditional U-slot patch, a SLR-fed patch without hairpin and the proposed filtering antenna. It is observed that the traditional patch antenna has two strong harmonics at 8.75 and $11.7 \mathrm{GHz}$. However, when the U-slot patch is fed and coupled by a SLR, the two harmonics are eliminated. This is attributed to the fact that the dual-mode SLR and the dual-band U-slot patch have the same fundamental resonant frequencies but different higher order harmonics. As a result, these two components are detuned at the high band and the higher order harmonics can be suppressed.

It should also be noted that the SLR itself also introduces an unwanted harmonic at $9.5 \mathrm{GHz}$. To overcome this interference, a hairpin resonator with its fundamental resonant frequency at $9.5 \mathrm{GHz}$ is shunted at the feed line, as presented in the proposed antenna. The hairpin resonator introduces a notch-band at 9.5 $\mathrm{GHz}$, which is used to eliminate the interference at that band. As a result, this antenna can achieve an excellent out-of-band rejection up to $12 \mathrm{GHz}$. Compared with the traditional method in [12], the harmonics are suppressed without increasing the footprint of the antenna. In addition, the frequency response, including the bandwidth and the frequency selectivity, are significantly improved.

\section{RESULTS AND DISCUSSION}

The simulated and measured $S_{11}$ of the proposed filtering antenna are presented in Fig. 5. A broad frequency range from 3

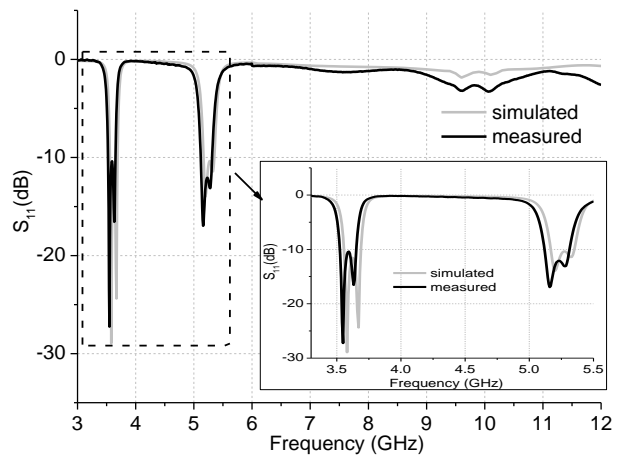

Fig. 5. Simulated and measured $S_{11}$ of the proposed dualband filtering patch antenna.

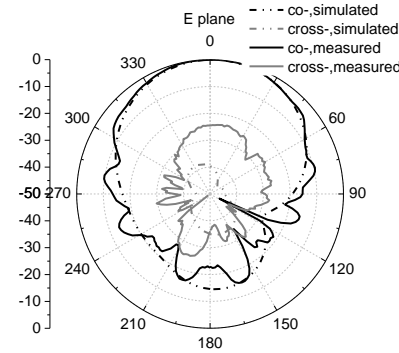

(a)

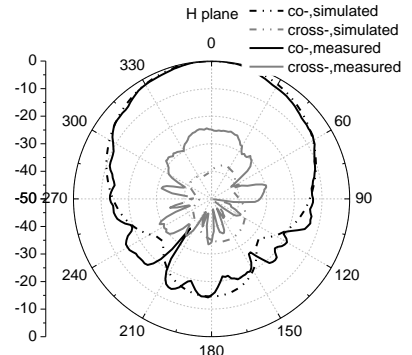

(b)
Fig. 6. The normalized simulated and measured radiation patterns at 3.6 GHz: (a) E plane, (b) H plane.

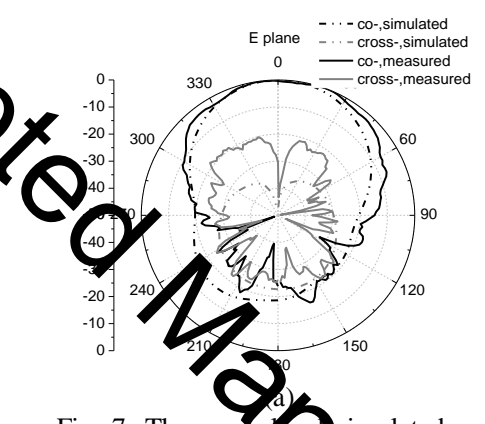

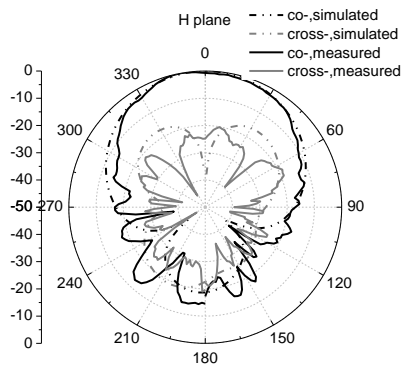

(b)
Fig. 7. The norma simulated and measured radiation patterns at 5.2 GHz: (a) E plane,

to $12 \mathrm{GHz}$ was tested show the harmonic suppression performance. The measure rasult agrees very well with the simulation with dual operation ands from 3.5 to $3.65 \mathrm{GHz}$ and 5.1 to $5.3 \mathrm{GHz}$ were achieved. The injor discrepancy between the simulation and the measuras attributed to the fabrication errors. At the both bands, $2^{\text {nd }}$-order response with two reflection zeros are identifiable. This results in improved bandwidth and filtering performance. Out of the bands, the antenna exhibits wideband harmonic suppression up to $12 \mathrm{GHz}$.

Fig. 6(a)-(b) shows the normalized simulated and measured co- and cross-polarization radiation patterns at $3.6 \mathrm{GHz}$ in the $\mathrm{E}$ (XOZ) and $\mathrm{H}$ plane (YOZ), respectively. The antenna exhibits a radiation in broadside direction with a cross polarization discrimination (XPD) of $-25 \mathrm{~dB}$ in both planes. The $\mathrm{E}$ and $\mathrm{H}$ plane radiation patterns at $5.2 \mathrm{GHz}$ are presented in Fig. 7. It is observed that the measured results agree well with simulations. Compared with the U-slot antenna in [3], the XPD of this antenna is significantly improved, especially in the directions offset the broadside. The discrepancy between the simulated and measured patterns, especially the nulls in the backward 


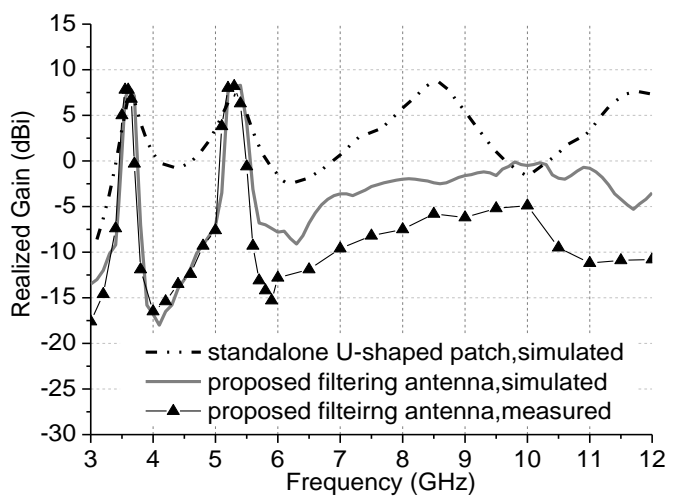

Fig. 8. The simulated and measured gain of the proposed dual-band filtering antenna.

\begin{tabular}{|c|c|c|c|}
\hline $\begin{array}{l}\text { Types of } \\
\text { antennas }\end{array}$ & $\begin{array}{l}\text { Harmonics } \\
\text { suppression }\end{array}$ & $\begin{array}{c}\text { Gain (dBi) } \\
\quad\left(f_{L} / f_{H}\right)\end{array}$ & $\begin{array}{c}\mathrm{XPD}(\mathrm{dB}) \\
\quad\left(f_{L} / f_{H}\right)\end{array}$ \\
\hline Ref. [13] & No & $-1.8 / 1.1$ & $-10 /-22$ \\
\hline Ref. [14] & No & $4.0 / 3.8$ & NA \\
\hline This work & Yes & $5 / 8.0$ & $-40 /-30$ \\
\hline
\end{tabular}

radiation, is mainly attributed to the influence of gasurement devices and the cables behind the antenna.

Fig. 8 shows the simulated and measured rean ed gains of the proposed dual-band filtering antenna from 3 to $A$. For comparison, the simulated gain of a standalone U-ster mh antenna is also included. The proposed antenna achieveth gains of 6.5 and $7 \mathrm{dBi}$ at low- and high-band, respectively the harmonics of 8.5 and $11.7 \mathrm{GHz}$, the traditional U-slot patch antenna has a gain of $7.5 \mathrm{dBi}$. However for the proposed filtering antenna, the gain drops sharply to below $-7.5 \mathrm{dBi}$ as the frequency offsets by $5.6 \%$ from the two central frequencies. At the two harmonic bands, the simulated and measured gains are significantly reduced to below -2 and $-5 \mathrm{dBi}$, respectively. This demonstrates that the proposed antenna has excellent frequency selectivity and out-of-band harmonic suppression over a wideband.

Table II compares the proposed dual-band filtering antenna with the other two reported dual-band filtering antennas in [13] and [14]. The comparison focuses on the harmonic suppression, polarization, gain and XPD at the two bands. This comparison shows that this work exhibits an improved gain and XPD at the two operation bands. The works in [13] and [14] lack the investigation of harmonic suppression and the gains are relatively lower than a traditional patch antenna. In addition, the design in [13] exhibits different polarizations at the two bands, and thus the technical contribution may be insufficient.

\section{CONCLUSION}

In this communication, a novel dual-band filtering antenna has been proposed by integrating a dual-mode resonator in the U-slot patch antenna design. The U-slot antenna as well as the resonator have been investigated. The proposed antenna exhibits an improved $2^{\text {nd }}$-order filtering response with two reflection zeros in both bands, which results in enhanced bandwidth and frequency selectivity. The bandwidth can be tuned by adjusting the coupling strength between the SLR and the patch. In addition, the harmonic suppression over a broadband has been studied. Simulated and measured results agree very well, showing an excellent frequency response in terms of impedance matching, bandwidth, frequency selectivity, out-of-band rejection, radiation and gain.

\section{REFERENCES}

[1] K. F. Lee, K. F. Tong, "Microstrip Patch antennas - Basic Characteristics and Some Recent Advances," IEEE Proceedings, vol. 100, no. 7, pp. 2169-2180, Jul. 2012.

[2] K. F. Lee, S. L. S. Yang, A. Kishk and K. M. Luk, "The Versatile U-slot Patch Antenna," IEEE Antennas and Propag. Magazine, vol. 52, no. 1, pp. 71-88, Feb. 2010.

[3] W. C. Mok, S. H. Woong, K. M. Luk, K. F. Lee, "Single-Layer Single Patch Dual-Band and Tri-Band Patch Antenna," IEEE Trans. Antennas and Propag., vol. 61, no. 8, pp. 4341-4344, Aug. 2013.

[4] I. Yeom, J. M. Kim and C. W. Jung, "Dual-Band Slot-Coupled Patch Antenna with Broad Bandwidth and High Directivity for WLAN Access Point," Electron. Lett., vol. 50, no. 10, pp. 726-728, 2014.

[5] Y. X. Guo, K. M. Luk and K. F. Lee, "Dual-Band Slot-Loaded Short-Circuited Patch Antenna," Electron. Lett., vol. 36, no. 4, pp. 289-291, 2000.

[6] M. Al-Joumayly, S. Aguilar, N. Behdad and S. Hagness, "Dual-Band Miniaturized Patch Antennas for Microwave Breast Imaging," IEEE Antenna Wireless Propag. Lett., vol. 9, pp. 268-271, 2010.

[7] S. Weigand, G. Huff, K. Pan and J. Bernhard, "Analysis and Design of Broad-Band Single-Layer Rectangular U-slot Microstrip Patch Antennas," IEEE Trans. Antennas and Propag., vol. 51, no. 3, pp. 457-468, Mar. 2003.

[8] C. X. Mao, S. Gao, Z. P. Wang, Y. Wang, F. Qin, B. Sanz and Q. X. Chu, "Integrated Filtering-Antenna with Controllable Frequency Bandwidth," $9^{\text {th }}$ European Conf. on Antenna and Propag., pp. 1-4, 2015.

[9] X. B. Shang, Y. Wang, W. L. Xia and M. Lancaster, "Novel Multiplexer Topologies Based on All-Resonator Structure," IEEE Trans. Microw. Theory Tech., vol. 61, no. 11, pp. 3838-3845, Nov. 2013.

(2) A. Abbaspour, J. Rizk, and G. Rebeiz, "Integration of filters and - Mrry strip antennas," in Proc. IEEE AP-S Int. Symp., 2002, pp. 874-877.

[11 W. Wu, Y. Z. Yin, S. L. Zuo, Z. Y. Zhang and J. J. Xie, "A New Comp Filter-Antenna for Modern Wireless Communication Systems," IEE A ten Wireless Propag. Lett., vol. 10, 2011, pp. 1131-1134.

[12] Y. J. Ken M. Farooqui and K. Chang, "A compact dual-frequency rectifyin 1 a with high-orders harmonic-rejection," IEEE Trans. Antennas an r. vol. 55, no. 7, pp. 2110-2113, Jul. 2007.

[13] L. Yang, P. Creop L. Han, W. W. Choi, K. W. Tam and K. Wu, "Miniaturized Paray Cl Goupled-Line Filter-Antenna With Spurious Response Suppressio i EEE Antennas and Wireless Propag. Lett., vol. 10 , no. , pp. 726-72 1

[14] C. Y. Hsieh, C. H. Wu and (G) Ma, "A Compact Dual-Band Filtering Patch Antenna Using Step Ennee Resonators," IEEE Antenna Wireless Propag. Lett., vol. 14, pp. J56-1059, 2015.

[15] Y. J. Lee, G. W. Cao and S. J. Chun, Compact Dual-Band Filtering Microstrip Antenna with the Sam aration Planes," in Proc. Asia-Pacific Microw. Conf., pp. 1178-11 0, 2012.

[16] K. Santasri, M. Debjani, "A planar microstrip-fed tri-band filtering antenna for WLAN/WiMAX applications," Microw. Opt. Technol. Lett., vol. 57, no. 1, pp. 233-237, 2015.

[17] X. Y. Zhang, J. X. Chen, Q. Xue and S. M. Li, "Dual-band Bandpass Filters Using Stub-Loaded Resonators," IEEE Microw. Wireless Components Lett., vol. 17, No. 8, pp. 583-585, Aug. 2007.

[18] X. Y. Zhang, C. H. Chan, Q. Xue and B. J. Hu, "Dual-Band Bandpass Filter with Controllable Bandwidths Using Two Coupling Patchs," IEEE Microw. Wireless Compon. Lett., vol. 20, no. 11, pp. 616-618, Nov. 2010.

[19] C. X. Mao, S. Gao, Y. Wang, F. Qin and Q. X. Chu, "Multi-mode resonator-fed dual polarized antenna array with enhanced bandwidth and selectivity," IEEE Trans. Antennas and Propag., vol. 63, no. 12, pp. 5492-5499, Dec. 2015. 